Disclosures N. Norris: None. I. Smith: None. C. Settanni: None. W. Merritt: None. T. Becker: 1; C; 2018 BAF Grant. 6; C; Significant Financial Interest in ATI.

\section{E-007 CHARACTERIZATION OF LIPOCALIN-2 IN ISCHEMIC STROKE BY DISTAL AND PROXIMAL INTRALUMINAL SAMPLING FROM MECHANICAL THROMBECTOMY}

${ }^{1} \mathrm{M}$ Sands*, ${ }^{1} \mathrm{~J}$ Frank, ${ }^{1} \mathrm{~B}$ Maglinger, ${ }^{2} \mathrm{~S}$ Grupke, ${ }^{3} \mathrm{~J}$ Fraser, ${ }^{1} \mathrm{~K}$ Pennypacker. 'University of Kentucky, lexington, $K Y_{;}{ }^{2}$ University of Kentucky Department of Neurology, University of Kentucky Department of Radiology, University of Kentucky, lexington, $K Y ;{ }^{3}$ University of Kentucky Department of Neurosurgery, University of Kentucky, Lexington, KY

\subsection{6/neurintsurg-2020-SNIS.43}

Introduction Lipocalin-2(LCN2) is a protein involved in many cellular processes, including the regulation of iron homeostasis, promotion of protective mechanisms in renal ischemia, and astrocyte activation in ischemic stroke. In some ischemic stroke models, LCN2 has been described as a 'help me' signal, leading to the activation of microglia, astrocytes, and drive towards cellular phenotypes favorable of recovery. While some models suggest pro-recovery effects of LCN2, other models illustrate LCN2 as having a critical role in neuroinflammation and reperfusion injury. The possibility of disease state-dependent influence of LCN2 activity in ischemic stroke has been recognized, but has yet to be further characterized.

Methods Human plasma samples from thrombectomy patients were processed in the Blood and Clot Thrombectomy Registry and Collaboration (BACTRAC clinicaltrials.gov NCT03153683), and underwent Proximity Extension Assay (PEA) via Olink (Olink Proteomics, Boston, MA). For each protein, intracranial expression distal to the stroke thrombus was compared to the same subject's systemic arterial blood as an internal control. The percent change of protein expression was calculated as follows: (intracranial distal values- systemic proximal values).Comorbidities and sex difference were analyzed using appropriate one-way comparisons and regression.

Results 25 adult patients ( $>18$ yrs) were included in this initial analysis, of which $15(60 \%)$ were female. Median age was 64 (24-91). 16 patients (64\%) had hypertension, 15 patients (60\%) had BMI >25, 10 patients (40\%) had a history of smoking, 6 patients $(24 \%)$ had previous stroke, 4 patients (16\%) had hyperlipidemia, and 1 patient (4\%) had previous MI. Mean infarct time was $513 \pm 246$ minutes and mean infarct volume was $58.17 \pm 82.28 \mathrm{cc}$. Of 184 proteins, only 27 demonstrated an increase percent change between intracranial and systemic blood. LCN2 demonstrated a 14\% increase change between intracranial and systemic blood, one of the greatest percent increases among measured proteins. The percent change of LCN2 was significantly increased in those with hypertension $(p=0.024)$ and decreased in those with type-two diabetes $(p=0.04)$.

Conclusions Changes of LCN2 intracranially during stroke were most significant in patients with hypertension and/or diabetes. For the first time, these data provide insight into the human molecular pathology of stroke regarding this protein and its signaling cascade. Future studies will focus on the role of proteins as they relate to radiographic, functional and other clinical outcomes. Proteomic findings coupled with advanced database analysis will elucidate complex cell signaling and biomolecular interactions that occur in the blood at the site of infarct.
Disclosures M. Sands: None. J. Frank: None. B. Maglinger: None. S. Grupke: None. J. Fraser: None. K. Pennypacker: None.

\section{E-008 A NOVEL LIQUID EMBOLIC MATERIAL USING A HYDROPHILIC POLYMER COMPOSITE ACTIVATED BY THE CA2+ IN THE BLOOD: ANGIOGRAPHICAL EVALUATION USING A RABBIT MODEL}

${ }^{1} \mathrm{Y} \mathrm{Yuki}{ }^{*},{ }^{2} \mathrm{~K}$ Ohkawa, ${ }^{1} \mathrm{~F} \mathrm{Hsu},{ }^{1} \mathrm{~J} \mathrm{Xu},{ }^{3} \mathrm{~S} \mathrm{Li},{ }^{1} \mathrm{~S}$ Suzuki. ${ }^{1}$ Neurosurgerey, UC Irvine Medical Center, Orange, $C A ;{ }^{2}$ Bioengineering, Institute for Fiber Engineering (IFES), Shinshu University, Nagano, Japan, Ueda-city, Nagano Prefecture, JAPAN; ${ }^{3}$ Surgery, UC Irvine Medical Center, Orange, CA

\subsection{6/neurintsurg-2020-SNIS.44}

Introduction Liquid embolic material (LEM) plays an essential role in the treatment of hemorrhagic stroke caused by arteriovenous malformation or dural arteriovenous fistula. However, currently available non-adhesive LEMs has the problem of catheter entrapment, and also known to have a cytotoxic effect due to the organic solvents such as Dimethyl Sulfoxide (DMSO). The New Generation Liquid Embolic Material (NGLEM) is a clear liquid that immediately forms a solid hydrogel cast upon exposure to $\mathrm{Ca} 2+$ in the bloodstream, and organic solvents are not required. The performance of this new liquid embolic material was evaluated using an in vivo experimental model using rabbit.

Methods Under general anesthesia, a renal artery of New Zealand rabbit $(4.5-5.0 \mathrm{~kg})$ was catheterized under fluoroscopy using a microcatheter, and NGLEM (Aqua Embolic System) was injected into the artery. Following factors were assessed; 1) the amount of LEM required for the complete occlusion, 2) injection speed, 3) duration of the injection, 4) radiopacity during the deployment and 5) incidence of catheter entrapment after the injection.

Results 10 renal arteries in 10 rabbits were treated, and all arteries were completely occluded without technical complication. The injected materials immediately formed LEM cast in all vessels followed by the reflux over the microcatheter. All catheters were withdrawn without any sign of catheter entrapment. The NGLEM mixed with tantalum based (10 animals) contrasts medium showed sufficient radiopacity under fluoroscopy. With the injection speed of $0.02 \mathrm{ml} / \mathrm{sec}$, the average volume required was $0.68 \mathrm{ml}$. Average time for the complete

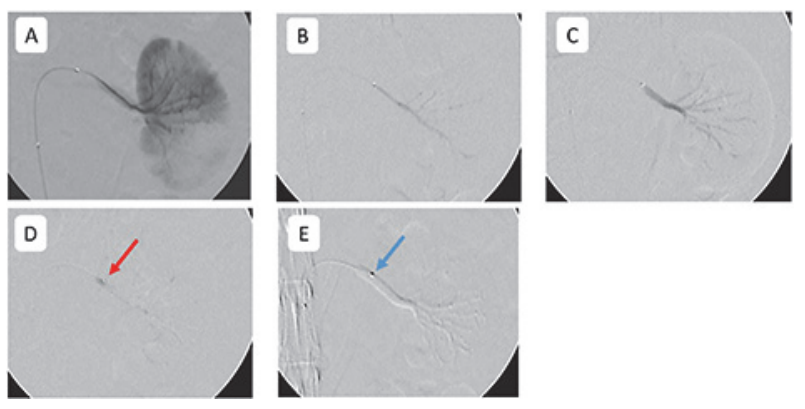

Abstract E-008 Figure 1 A left Renal artery of rabbit was embolized with Aqua Embolic System. A) A control angiogram was performed. B) and C) The material injected from the catheter filling the distal branches. D) a reflux of the material (red arrow) was seen. E) At the end of the procedure, the tip of the catheter was embedded in the cast of embolic material (blue arrow), which was removed without any resistance 
occlusion was 237 seconds. No increased thrombogenicity or vasospasm near the treated lesion was observed during the procedure.

Conclusions NGLEM, which is a DMSO free, non-adhesive bio-polymer may be used as an embolic material for the treatment of hemorrhagic stroke caused by cerebrovascular diseases.

Disclosures I. Yuki: 1; C; Research Grant: Institute for Clinical and Translational Science (ICTS) Pilot Award (NIH Director's Transformative Research Award initiative), Research Grant Supported by the MEXT (Ministry of Education, Culture, Sports, Science and Technology), Government of Japan. K. Ohkawa: 1; C; Research Grant Supported by the MEXT (Ministry of Education, Culture, Sports, Science and Technology), Government of Japan. 2; C; N/A. 3; C; N/A. 4; C; N/ A. 5; C; N/A. 6; C; N/A. F. Hsu: 1; C; Research Grant: Institute for Clinical and Translational Science (ICTS) Pilot Award. J. Xu: None. S. Li: None. S. Suzuki: 1; C; Research Grant: Institute for Clinical and Translational Science (ICTS) Pilot Award (NIH Director's Transformative Research Award initiative).

\section{E-009 A PROSPECTIVE, MULTICENTER REGISTRY FOR THE INVESTIGATION OF CLOT IN ISCHEMIC STROKE AND HEMATOMA EVACUATION: STUDY PROTOCOL FOR INSIGHT}

${ }^{1} \mathrm{C}$ Kellner*, ${ }^{2} \mathrm{~J}$ Fraser. ${ }^{1}$ Dept. of Neurosurgery, Mount Sinai Health System, New York, NY; ${ }^{2}$ Departments of Neurosurgery, Neurology, Radiology, and Neuroscience, University of Kentucky, Lexington, $K Y$

\subsection{6/neurintsurg-2020-SNIS.45}

Introduction/Purpose Multiple clinical studies have established the importance of effective clot evacuation on good clinical outcomes for acute ischemic and hemorrhagic stroke, and suggest that thrombus composition can impact treatment efficacy. Understanding clot composition and associated genomic, epigenomic, and proteomic signatures could provide insight into thrombus biology, etiology, and aid in prognosis. The primary objective of the INSIGHT study is to develop a 'multi-omic' blood clot signature to predict the etiology of the blood clot in patients with ischemic stroke or intracerebral hemorrhage $(\mathrm{ICH})$. Clots retrieved through either mechanical thrombectomy or endoscopic ICH aspiration will be analyzed with histology, RNA sequencing, and proteomics while contemporaneously collected peripheral blood samples will undergo single nucleotide polymorphism (SNP) analysis, RNA sequencing, and proteomic analysis. A secondary objective is to identify specimen characteristics that correlate with patient clinical presentation, procedural outcome, hospital length of stay, and 90-day functional outcome.

Materials and Methods INSIGHT is a prospective, multicenter registry for the collection and analysis of specimens collected from ischemic stroke thrombectomy procedures and minimally invasive interventions for intracerebral hematoma evacuation utilizing the Penumbra Aspiration Pump. The registry will enroll up to 1000 patients from up to 50 U.S. sites. Patients age $\geq 18$ years, treated frontline with the Penumbra System (for ischemic stroke patients eligible for mechanical thrombectomy) or the Artemis Neuro Evacuation device (for intracerebral hematoma evacuation in patients eligible for minimally invasive surgery), and with extracted thrombus/ embolus, will be included. Exclusion criteria includes current participation in a clinical trial that may confound the ability to capture clot and/or influence clot composition. Patient demographics, medical history, procedural information, and extracted clot and blood specimen will be collected. Followup assessments will occur at discharge and at 90 days postprocedure.

Results The estimated date for study completion is 2023.

Conclusion We report the design of the INSIGHT study, a prospective 'multi-omic' registry to analyze specimen collected during ischemic stroke thrombectomy and minimally invasive intracerebral hematoma evacuation.

Disclosures C. Kellner: None. J. Fraser: None.

\section{E-010 INVESTIGATION OF A NOVEL POLY(PROPYLENE GLYCOL) MATERIAL FOR USE AS A PROTEIN-RESISTANT, BIO-INERT IMPLANT}

${ }^{1} \mathrm{~W}$ Merritt*, ${ }^{2} \mathrm{~A}$ Koppisch, ${ }^{2} \mathrm{R}$ Kellar, ${ }^{1} \mathrm{~A}$ Ducruet, ${ }^{1} \mathrm{~T}$ Becker. ${ }^{1}$ Bioengineering Program, Northern Arizona University, Flagstaff, AZ; ${ }^{2}$ Biology, Northern Arizona University, Flagstaff, $A Z$

\subsection{6/neurintsurg-2020-SNIS.46}

Introduction/Purpose A poly(propylene glycol)-based material (PPODA-QT) is currently being investigated as a liquid embolicfor the treatment of intracranial aneurysms. Initial biocompatibility results have shown that PPODA-QTis uniquely bioinert due to its ability to resist protein adsorption from contact with blood. Preliminaryanimal implant studies of PPODAQT in rabbits (1- and 3-month survivals) and canines (6month survival)have shown a lack of protein adsorption, lack of foreign body response, and minimal encapsulationaround the material. A protein-resistant, liquid-to-solid curing material could have expanded use as anon-fouling, protein-resistant coating for a variety of metal-based implants.

Materials and Methods The resistance to protein adsorption is quantified via protein depletion from the blood, as well asthrough analysis of desorbed proteins from sample surfaces via sodium dodecyl sulphate (SDS).Detection and quantification of proteins is performed via PPODA-QT samples $(n=12)$ were prepared andcured in $4 \mathrm{~mm}$ diameter cylindrical molds with a height of $10 \mathrm{~mm}$. PPODA-QT samples were eachimmersed in $1.5 \mathrm{~mL}$ of heparinized rabbit whole blood within a $2 \mathrm{~mL}$ polypropylene vial. Vials were placedon a shaker plate for 15 minutes to allow for ensure maximal interfacing between blood and thesamples. With the majority of protein adsorption happening within seconds, 15 minutes is sufficient forprotein adsorption. Positive controls $(n=4)$ were created by preparing $4 \mathrm{~mm}$ diameter and $10 \mathrm{~mm}$ thickpolyurethane cylinders and subjecting them to the same blood immersion procedure. Negative controls $(n=4)$ were created by filling vials with blood and no sample to give a baseline level for proteinadsorption onto the vials themselves. Proteins will be quantified via a protein quantitation assay thatutilizes a fluorescent dye to tag proteins for analysis with a spectrofluorometer with sensitivity as low as $10 \mathrm{ng} / \mathrm{mL}$.

Results PPODA-QT has been shown to exhibit exemplary protein-resistant properties as well as minimalencapsulation and inflammatory response when implanted while providing a relatively uniform surfacefor neointimal tissue growth across the device at the neck of the aneurysm. Comparison 